\title{
Left Atrial Intramural Hematoma after Catheter Ablation for Atrial Tachyarrhythmia
}

Myung-jin Cha, MD'; Seil Oh, MD, PhD, FHRS $^{2}$

${ }^{1}$ Healthcare System Gangnam Center and ${ }^{2}$ Department of Internal Medicine, Seoul National University Hospital, Seoul, Korea

Received: January 14, 2015

Revision Received: May 30, 2015

Accepted: June 29, 2015

Correspondence: Seil Oh, MD, PhD, FHRS,

Professor of Internal Medicine, Seoul National

University College of Medicine and Seoul

National University Hospital, 101 Daehak-ro,

Jongno-gu, Seoul 110-744, Korea

Tel: +82-2-2072-2088, Fax: +82-2-762-9662

E-mail: seil@snu.ac.kr

Copyright (C) 2015 The Official Journal of Korean Heart Rhythm Society Editorial Board \& MMK Co., Ltd.

\begin{abstract}
We report the rare case of a 70-year-old man with a large intramural hematoma associated with radiofrequency catheter ablation for left side atrial tachyarrhythmias. The size of the hematoma increased, and it compromised mitral inflow within a day. Emergent open cardiac surgery was performed for hematoma evacuation and Cox-Maze procedure. He had no immediate postoperative complications and was free of tachyarrhythmia over a 1-year follow-up.
\end{abstract}

Key Words: - Radiofrequency Catheter Ablation - complications - Hematoma

\section{Introduction}

Radiofrequency catheter ablation (RFCA) is an established and effective treatment method for various atrial tachyarrhythmias. Although a thromboembolism has been widely reported as the principle complication of conventional RFCA, bleeding complications, including intramural hematomas, have been less often reported. ${ }^{1}$ Intramural atrial hematomas are rare, but they have been described as occurring both spontaneously ${ }^{2}$ and iatrogenically., ${ }^{3.4}$ Here we report the case of a patient with an intramural hematoma associated with RFCA for intractable atrial tachyarrhythmias, who was treated with open cardiac surgery.

\section{Case}

A 70-year-old man with a 3-year history of medically intractable paroxysmal atrial tachycardia (AT) and paroxysmal atrial fibrillation $(\mathrm{AF})$ with symptoms of severe palpitation and near-syncope was admitted to the Arrhythmia Center of Seoul National University Hospital.

He had hypertension and was taking an angiotensin-receptor blocker, a beta-blocker, amiodarone, and warfarin. His palpitation continued in spite of treatment with various antiarrhythmic agents, and amiodarone-associated hypothyroidism was suspected. Several months previously, his symptoms worsened compared to those experienced earlier. He visited the emergency department twice within a 4-month period, and cardioversion was performed at every visit. Figures 1A-1D show his various types of atrial tachyarrhythmias.

An electrophysiological study revealed that the atrial tachycardias originated from six different focal sites. Catheter ablation was performed with an electroanatomical mapping system (CARTO-3, Biosense) at the area of the vein of Marshall, left atrial (LA) septum, and cavotricuspid isthmus. 
A

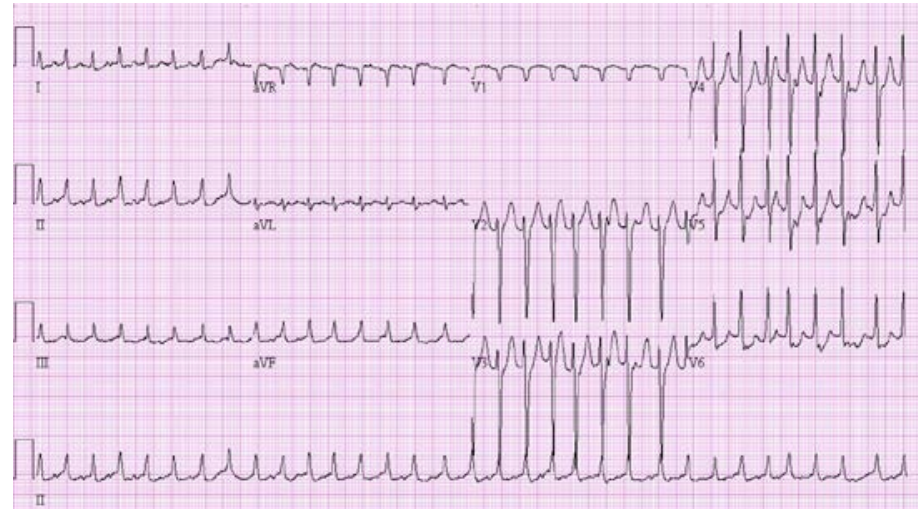

B

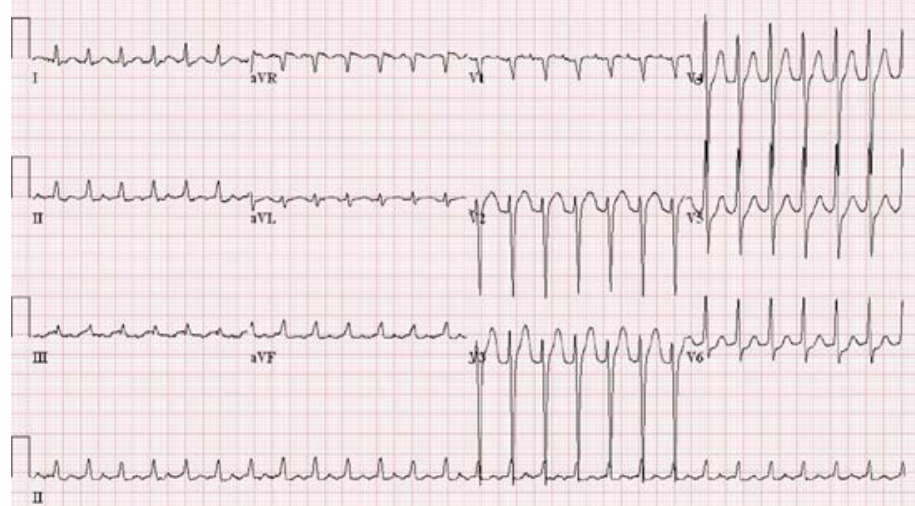

C

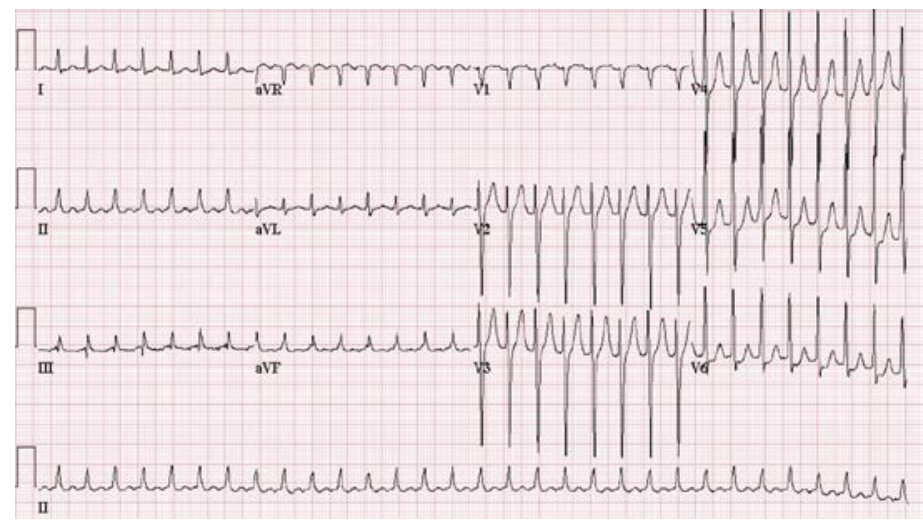

D

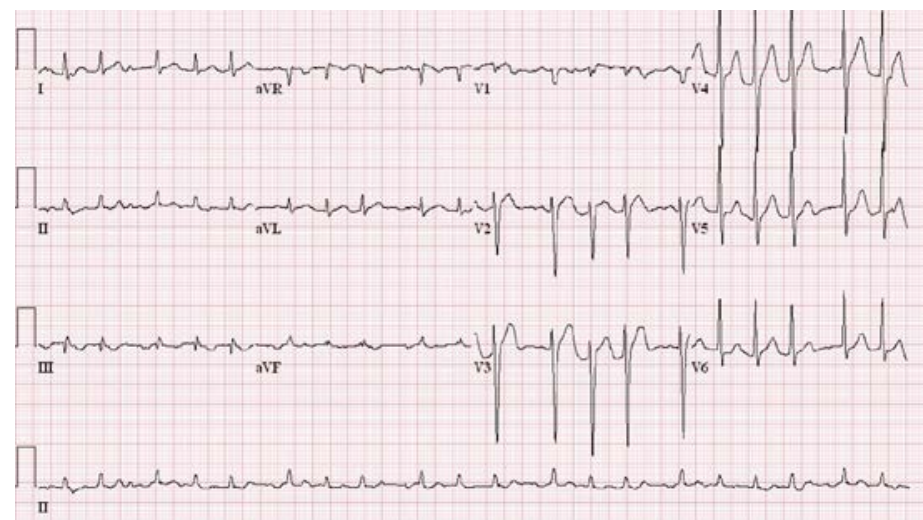

Figure 1. The patient's various types of atrial tachyarrhythmias. (A)-(C) different types of atrial tachycardias. (D) atrial fibrillation in the patient. 


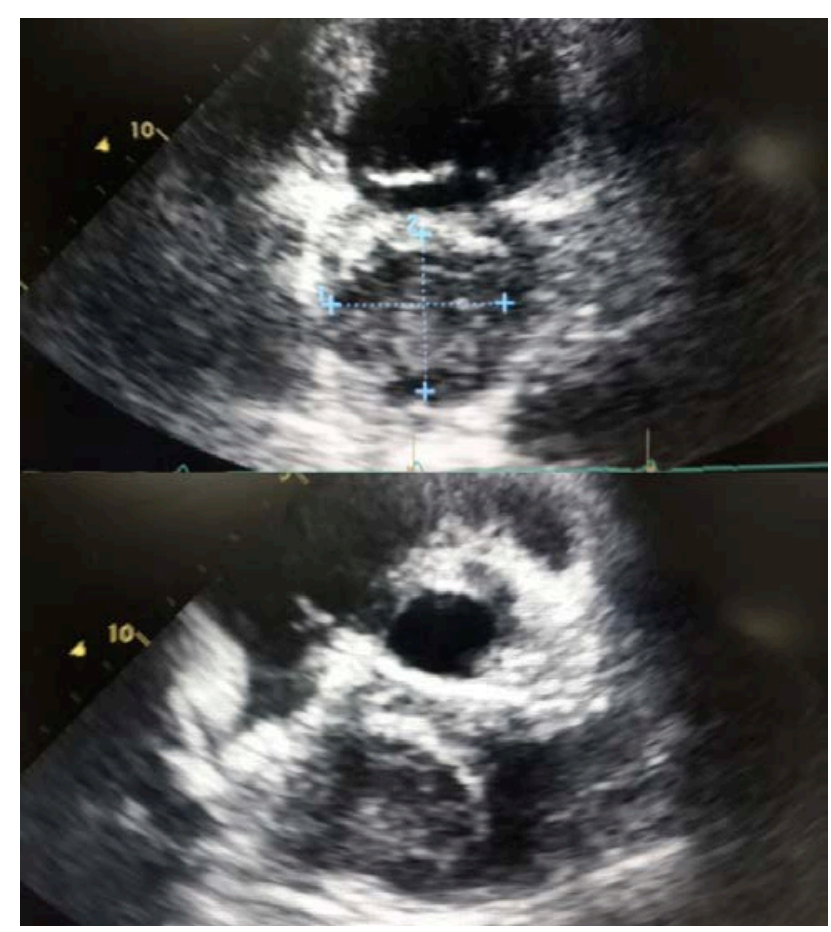

Figure 2. An echocardiographic image of the intramural hematoma. A $3.5 \times$ 4.2-cm sized hyperechogenic, smoothly contoured, round immobile mass is seen at the left atrial posterior wall near the interatrial septum.

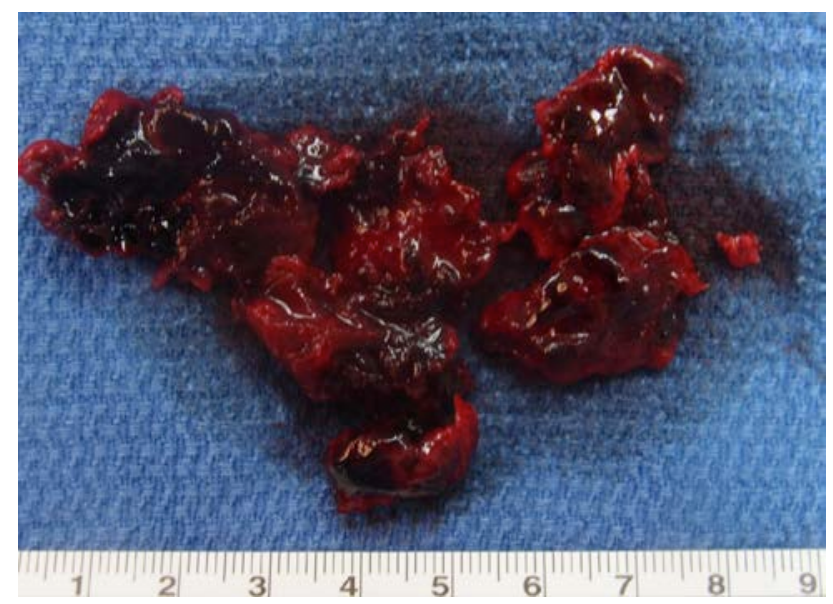

Figure 3. A gross image of the evacuated intramural hematoma. An unorganized hematoma, which was surgically suctioned out, is seen.

Circumferential antral ablation was performed on four pulmonary veins, including the carina, for AF ablation. After RFCA for AF and AT, three types of focal ATs were sustained and continuously changing from one type to another. Thus, DC cardioversion ( $5 \mathrm{~J}$, internal) was performed to convert to sinus rhythm.

After the first RFCA, he visited the emergency department four times within a 5-month period, and cardioversion was performed at three visits. Five months later, he was admitted for a second RFCA procedure. He arrived at the EP laboratory with a sinus rhythm. Systemic anticoagulation with intravenous heparin was initiated immediately after transseptal puncture. Activated clotting time (ACT) was checked every 30 minute to ensure it remained at 300-350 s.

Three different ATs were easily induced with programmed electrical stimulation. A three dimensional activation map was prepared using the CARTO-3 (Biosense) mapping system. Ablation was performed using a $7.5 \mathrm{~F}$ open irrigation catheter (NAVISTAR; Biosense Webster; DF curve, SF). Ablations were performed at the LA posterior area, posterior line, roof line, superior vena cava to foramen ovale line, right atrial (RA) ant wall, inferoseptal septal area of LA, LA low septum, and mid-coronary sinus area. After 9 hours of partially successful ablations, DC cardioversion ( $5 \mathrm{~J}$, internal) was performed to convert to sinus rhythm. An immediate post-procedure echocardiography showed no apparent procedure-related complications.

Routine echocardiography was performed at 3 hours after the procedure, and a $3.5 \times 4.2 \mathrm{~cm}$-sized hyperechogenic, smoothly contoured, round immobile mass was detected at the left atrial posterior wall near the interatrial septum, without flow obstruction (Figure 2). Echocardiography performed the following day showed an increase in size to $3.9 \times 4.9 \mathrm{~cm}$ with partial flow obstruction, and emergent open cardiac surgery was performed for hematoma evacuation and Cox-Maze procedure.

The LA was approached by extending an incision from the RAtomy for Maze to the posterior RA appendage and opening the LA roof. The posterior wall of the LA roof was swollen, and it almost filled the entire left atrium. However, no tear or puncture was found on the internal wall of the left atrium, and the wall color was normal. Needle aspiration of the posterior wall of the swollen LA was performed with no initial result. However, when the opening was enlarged, an unorganized hematoma was suctioned out. A 4-cm incision was made at the opening, and a hematoma was evacuated using a suction tip and forceps until the outer wall of the LA could be visualized (Figure 3). The patient recovered without any immediate postoperative complications.

Over a 1-year follow-up, no recurrences of AT or AF were noted, and the patient maintained sinus rhythm. 


\section{Discussion}

RFCA is typically performed through a percutaneous approach under fluoroscopic guidance. The incidence of systemic thromboembolic complications associated with RFCA in the left heart has been reported to be as high as $1-5 \%$, but bleeding complications, such as intramural hematoma, are rare..$^{5-7} \mathrm{An}$ intramural hematoma can be diagnosed using various imaging modalities, including echocardiography, cardiac computed tomography, and cardiac magnetic resonance imaging, which show a non-enhancing mass within the wall of the atrium.

The findings in the present case suggest that optimal periprocedural anticoagulation with heparin might reduce the risk of procedure related thromboembolic complications, but physicians should always pay attention to the risk of bleeding. Post-procedural imaging studies and short-term follow-up could help in the early detection and prevention of fatal bleeding complications.

\section{References}

1) Zhou L, Keane D, Reed G, Ruskin J. Thromboembolic complications of cardiac radiofrequency catheter ablation: a review of the reported incidence, pathogenesis and current research directions. J Cardiovasc Electrophysiol. 1999;10:611-620.

2) Shaikh N, Rehman NU, Salazar MF, Grodman RS. Spontaneous intramural atrial hematoma presenting as a left atrial mass. J Am Soc Echocardiogr. 1999;12:1101-1103.

3) Sah R, Epstein LM, Kwong RY. Images in cardiovascular medicine. Intramural atrial hematoma after catheter ablation for atrial tachyarrhythmias. Circulation. 2007;115:e446-447.

4) Kelly S, Bicknell SG, Sharma S. Left atrial wall hematoma after radiofrequency ablation for atrial fibrillation. AJR Am J Roentgenol. 2006;186:1317-1319.

5) Cappato R, Calkins H, Chen SA, Davies W, Iesaka Y, Kalman J, Kim YH, Klein G, Packer D,Skanes A. Worldwide survey on the methods, efficacy, and safety of catheter ablation for human atrial fibrillation. Circulation. 2005;111:1100-1105.

6) Cappato R, Calkins H, Chen SA, Davies W, Iesaka Y, Kalman J, Kim YH, Klein G, Natale A, Packer D, Skanes A. Prevalence and causes of fatal outcome in catheter ablation of atrial fibrillation. $J$
Am Coll Cardiol. 2009;53:1798-1803.

7) Bertaglia E, Zoppo F, Tondo C, Colella A, Mantovan R, Senatore G, Bottoni N, Carreras G, Coro L, Turco P, Mantica M, Stabile G. Early complications of pulmonary vein catheter ablation for atrial fibrillation: a multicenter prospective registry on procedural safety. Heart Rhythm. 2007;4:1265-1271. 